\title{
Search for Solar Axions: CAST
}

Biljana Lakić $^{* 1}$, S. Andriamonje ${ }^{3}$, V. Arsov ${ }^{14}$, S. Aune ${ }^{3}$, D. Autiero ${ }^{2, a}$, F. Avignone ${ }^{4}$, K. Barth ${ }^{2}$, A. Belov ${ }^{12}$, B. Beltrán ${ }^{7}$, H. Bräuninger ${ }^{6}$, J.M. Carmona ${ }^{7}$, S. Cebrián ${ }^{7}$, E. Chesi ${ }^{2}$, J.I. Collar ${ }^{8}$, R. Creswick ${ }^{4}$, T. Dafni ${ }^{5, b}$, M. Davenport ${ }^{2}$, L. Di Lella ${ }^{2, c}$, C. Eleftheriadis ${ }^{6}$, J. Englhauser 6 , G. Fanourakis ${ }^{10}$, H. Farach ${ }^{4}$, E. Ferrer ${ }^{3}$, H. Fischer ${ }^{11}$, J. Franz ${ }^{11}$, P. Friedrich ${ }^{6}$, T. Geralis ${ }^{10}$, I. Giomataris ${ }^{3}$, S. Gninenko ${ }^{12}$, N. Goloubev ${ }^{12}$, R. Hartmann ${ }^{6}$, M.D. Hasinoff ${ }^{13}$, F.H. Heinsius ${ }^{11}$, D.H.H. Hoffmann ${ }^{5}$, I.G. Irastorza ${ }^{3}$, J. Jacoby ${ }^{14}$, D. Kang ${ }^{11}$, K. Königsmann ${ }^{11}$, R. Kotthaus ${ }^{15}$, M. Krčmar ${ }^{1}$, K. Kousouris ${ }^{10}$, M. Kuster ${ }^{5,6}$, C. Lasseur ${ }^{2}$, A. Liolios ${ }^{9}$, A. Ljubičić ${ }^{1}$, G. Lutz ${ }^{15}$, G. Luzón ${ }^{7}$, D.W. Miller, A. Morales ${ }^{7, d}$, J. Morales ${ }^{7}$, A. Ortiz ${ }^{7}$, T. Papaevangelou ${ }^{2}$, A. Placci ${ }^{2}$, G. Raffelt ${ }^{15}$, J. Ruz ${ }^{7}$, H. Riege ${ }^{5}$, M.L. Sarsa ${ }^{7}$, I. Savvidis ${ }^{9}$, P. Serpico ${ }^{15}$, Y. Semertzidis ${ }^{5, e}$, L. Stewart ${ }^{2}$, J.D. Vieira ${ }^{8}$, J. Villar ${ }^{7}$, L. Walckiers ${ }^{2}$, K. Zachariadou ${ }^{10}$ and $\mathrm{K}$. Zioutas ${ }^{16}$

${ }^{1}$ Rudjer Bošković Institute, Zagreb, Croatia

Email: Biljana.Lakic@cern.ch

${ }^{2}$ European Organization for Nuclear Research (CERN), Geneve, Switzerland

${ }^{3}$ DAPNIA, Centre d'Études Nucléaires de Saclay (CEA-Saclay), Gif-sur-Yvette, France

${ }^{4}$ Department of Physics and Astronomy, University of South Carolina, Columbia, SC, USA

${ }^{5}$ GSI-Darmstadt and Institut für Kernphysik, TU Darmstadt, Darmstadt, Germany

${ }^{6}$ Max-Planck-Institut für extraterrestrische Physik, Garching, Germany

${ }^{7}$ Instituto de Física Nuclear y Altas Energías, Universidad de Zaragoza, Zaragoza, Spain

${ }^{8}$ Enrico Fermi Institute and KICP, University of Chicago, Chicago, IL, USA

${ }^{9}$ Aristotle University of Thessaloniki, Thessaloniki, Greece

${ }^{10}$ National Center for Scientific Research 'Demokritos', Athens, Greece

${ }^{11}$ Albert-Ludwigs-Universität Freiburg, Freiburg, Germany

${ }^{12}$ Institute for Nuclear Research (INR), Russian Academy of Sciences, Moscow, Russia

${ }^{13}$ Department of Physics and Astronomy, University of British Columbia, Vancouver, Canada

${ }^{14}$ Johann Wolfgang Goethe-Univ., Inst. für Angewandte Physik, Frankfurt am Main, Germany

${ }^{15}$ Max-Planck-Institut für Physik (Werner-Heisenberg-Institut), Munich, Germany

${ }^{16}$ Physics Department, University of Patras, Greece

The CERN Axion Solar Telescope (CAST) is searching for axions produced in the Sun's core by the Primakoff process. CAST is using a decommissioned Large Hadron Collider (LHC) test magnet where axions could be converted back into X-rays with energies up to $10 \mathrm{keV}$. Analysis of the 2003 data showed no signal above background implying an upper limit for the axion-photon coupling constant $g_{a \gamma \gamma}<1.16 \times 10^{-10} \mathrm{GeV}^{-1}$ at $95 \%$ C.L. for $m_{a} \lesssim 0.02 \mathrm{eV}$ [1]. The higher quality 2004 data is presently under analysis. CAST Phase II is scheduled to start in late 2005. This will be the first step in extending CAST's sensitivity to axion rest masses up to $\sim 1 \mathrm{eV}$.

International Europhysics Conference on High Energy Physics

July 21st - 27th 2005

Lisboa, Portugal

${ }^{*}$ Speaker. 


\section{Introduction}

Axions are pseudoscalars arising in models which resolve the strong CP problem in quantum chromodynamics (QCD) by the Peccei-Quinn (PQ) mechanism [2]. Owing to their potential abundance in the early universe, they are also well-motivated dark matter candidates. Axion phenomenology is determined by the scale $f_{\mathrm{PQ}}$ of the PQ symmetry breaking. The axion mass is given by the relation $m_{\mathrm{PQ}}=6 \mathrm{eV}\left(10^{6} \mathrm{GeV} / f_{\mathrm{PQ}}\right)$. Most of the axion experimental searches rely on the axion interaction with two photons with the coupling constant $g_{\text {arr }}=\alpha(E / N-1.92 \pm 0.08) / 2 \pi f_{\mathrm{PQ}}$, where $E / N$ is a model-dependent parameter. As a consequence, axions could transform into photons and vice versa in external electric or magnetic fields - the Primakoff process. Stars could produce axions by transforming thermal photons in the Coulomb fields of nuclei and electrons in the stellar plasma. The nearest and brightest source of axions would be the Sun. The expected solar

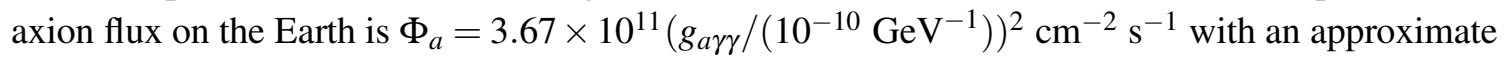
spectrum $^{1}$

$$
\frac{d \Phi_{a}}{d E_{a}}=3.821 \times 10^{10}\left(\frac{g_{a \gamma \gamma}}{10^{-10} \mathrm{GeV}^{-1}}\right)^{2} \frac{\left(E_{a} / \mathrm{keV}\right)^{3}}{e^{E_{a} / 1.103 \mathrm{keV}}-1} \mathrm{~cm}^{-2} \mathrm{~s}^{-1} \mathrm{keV}^{-1}
$$

and an average energy of $4.2 \mathrm{keV}$. Axions could be back-converted into X-rays in a laboratory magnetic field [3]. The expected number of X-rays reaching a detector is $N_{\gamma}=\int\left(d \Phi_{a} / d E_{a}\right) P_{a \rightarrow \gamma} S t d E_{a}$ where $P_{a \rightarrow \gamma}$ is the axion-photon conversion probability, $S$ is the effective area and $t$ is the measurement time (for example, $N_{\gamma} \approx 30$ for $g_{a \gamma \gamma}=10^{-10} \mathrm{GeV}^{-1}, S=14.5 \mathrm{~cm}^{2}$ and $t=100 \mathrm{~h}$ ). The conversion probability in vacuum is $P_{a \rightarrow \gamma}=\left(g_{a \gamma \gamma} B / q\right)^{2} \sin ^{2}(q L)$ where $L$ is the path length, $B$ is the magnetic field and $q=m_{a}^{2} / 2 E_{a}$ is the axion-photon momentum difference. The coherence condition, $q L<\pi$, restricts experimental sensitivity to a range of axion rest masses. For example, the coherence length of $L=10 \mathrm{~m}$ in vacuum requires $m_{a} \lesssim 0.02 \mathrm{eV}$ for $E_{a}=4.2 \mathrm{keV}$. The coherence can be restored for higher axion rest masses with the presence of a buffer gas which would provide an effective photon mass $m_{\gamma}$ so that $q=\left|m_{\gamma}^{2}-m_{a}^{2}\right| / 2 E_{a}$. As a result, the coherence would be restored for a narrow mass window around $m_{a}=m_{\gamma}[4]$.

After the first proposal of the axion helioscope principle [3], the first implementation was performed in [7]. Most recently, the Tokyo axion helioscope [8] of $L=2.3 \mathrm{~m}$ and $B=3.9 \mathrm{~T}$ has provided the limit $g_{a \gamma \gamma}<6.0 \times 10^{-10} \mathrm{GeV}^{-1}$ at $95 \%$ C.L. for $m_{a} \lesssim 0.03 \mathrm{eV}$ (vacuum) and $g_{a \gamma \gamma}<6.8-10.9 \times 10^{-10} \mathrm{GeV}^{-1}$ for $m_{a} \lesssim 0.3 \mathrm{eV}$ (variable-pressure buffer gas)[9]. Limits from crystal detectors $[10,11,12]$ are much less restrictive.

\section{CAST experiment}

The external magnetic field in the case of CAST is provided by a decomissioned LHC prototype dipole magnet [13] with $B=9.0 \mathrm{~T}$ and $L=9.26 \mathrm{~m}$. The magnet has two paralell, straight pipes with $S=2 \times 14.5 \mathrm{~cm}^{2}$ cross-sectional area. It is mounted on a platform with $\pm 8^{\circ}$ vertical and $\pm 40^{\circ}$ horizontal movement, allowing for observation of the Sun for $1.5 \mathrm{~h}$ at both sunrise and sunset during the whole year. The time the Sun is not reachable is devoted to background measurements.

\footnotetext{
${ }^{1}$ The spectrum in [4] has been changed to that proposed in [5], however with a modified normalization constant to match the total axion flux used here, which is predicted by a more recent solar model [6].
} 
A full cryogenic station is used to cool the superconducting magnet down to $1.8 \mathrm{~K}$. At both ends of the magnet, three different detectors are searching for X-rays coming from axion conversion in the magnet when it is pointing to the Sun. As X-ray detectors CAST utilizes: an X-ray mirror telescope in combination with a Charge Coupled Device (CCD), a conventional Time Projection Chamber (TPC) and a smaller gaseous chamber with novel MICROMEGAS (micromesh gaseous structure - MM) readout. The X-ray focusing system and MM are looking for sunrise axions, while the TPC is occupying both bores on the other end and is waiting for sunset axions. The X-ray mirror telescope can focus the photons coming from axion conversion to a $\sim 6 \mathrm{~mm}^{2}$ spot, thus enhancing the signal-to-background ratio and significantly improving the sensitivity of the experiment.

\section{First results and prospects}

The operation of the CAST experiment is foreseen to go in two phases:

- Phase I: during 2003 and 2004 the experiment operated with vacuum inside the magnet pipes and explored the axion mass range up to $0.02 \mathrm{eV}$. The 2003 data have been analyzed [1]. No signal above background was observed, implying an upper limit to the axion-photon coupling constant $g_{a \gamma \gamma}<1.16 \times 10^{-10} \mathrm{GeV}^{-1}$ (figure 1). During 2004, higher quality data were obtained (optimal performances of the detectors, lower background). The analysis is under way.

- Phase II: in order to extend CAST sensitivity to higher axion rest masses, magnet pipes will be filled with a buffer gas (first ${ }^{4} \mathrm{He}$ and later ${ }^{3} \mathrm{He}$ ). Gas pressure will be increased from 0 to 60 mbar (or more) in appropriate steps to cover all masses up to $\sim 1 \mathrm{eV}$. Data taking is expected to start in late 2005 and will continue in 2006 and 2007. The predicted limit on the axion-photon coupling constant is shown in figure 1.

Apart from this main line of research, CAST could also be sensitive to high energy axions (for example, axions from nuclear transitions in the Sun) and Kaluza-Klein axions [14].

\section{Conclusions}

The limit from the 2003 data improves the best previous laboratory result [8] on the axionphoton coupling constant by a factor 5 in our coherence region $m_{a} \lesssim 0.02 \mathrm{eV}$. It is also comparable, in this range of masses, to the astrophysical limit, $g_{a \gamma \gamma}<10^{-10} \mathrm{GeV}^{-10}$, derived from stellar energy-loss arguments [15]. CAST Phase II, with variable gas pressure inside the magnet pipes, will start at the end of 2005. The sensitivity extended to higher axion rest masses will allow us to enter the theoretically favoured region ("Axion models" in figure 1).

We acknowledge support from MSES (Croatia) and partial support by the European Union under the Ilias project, contract no. RII3-CT-2004-506222.

\footnotetext{
${ }^{a}$ Present address: Inst. de Physique Nucléaire, Lyon, France

${ }^{b}$ Present address: DAPNIA, CEA-Saclay, Gif-sur-Yvette, France

${ }^{c}$ Present address: Scuola Normale Superiore, Pisa, Italy

${ }^{d}$ Deceased

e Permanent address: Brookhaven National Laboratory, NY-USA
} 


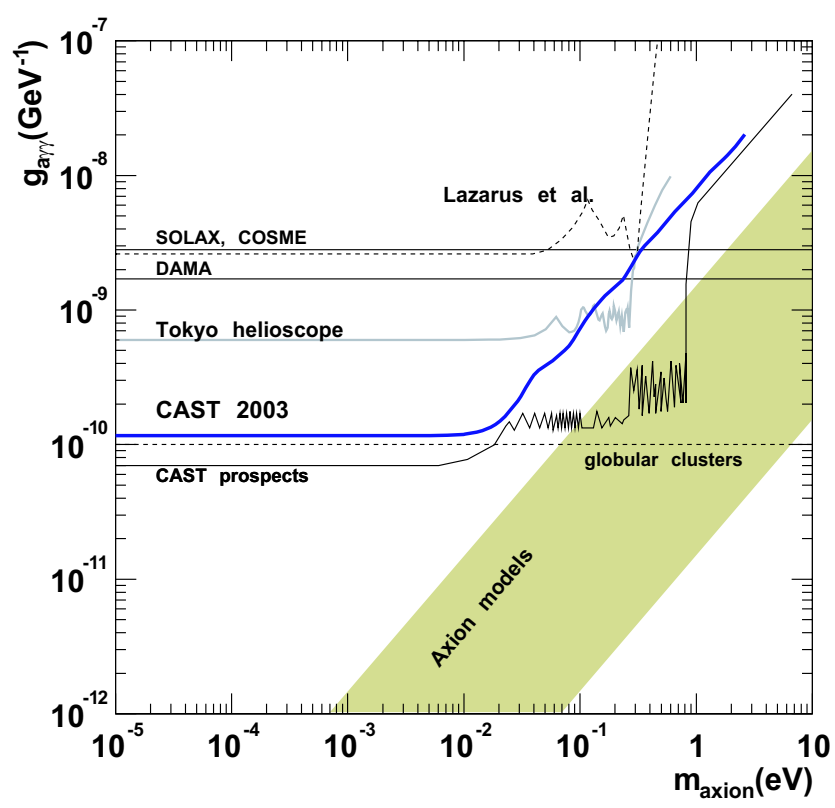

Figure 1: Exclusion limit (95\% C.L.) from the CAST 2003 data compared with constraints from other experiments. Also shown is the future CAST sensitivity as foreseen in the experiment proposal. The shaded band represents theoretically favoured region.

\section{References}

[1] K. Zioutas et al., Phys. Rev. Lett. 94, 121301 (2005).

[2] R. D. Peccei and H. R. Quinn, Phys. Rev. Lett. 38, 1440 (1977); Phys. Rev. D16, 1791 (1977).

[3] P. Sikivie, Phys. Rev. Lett. 51, 1415 (1983) [Erratum-ibid. 52, 695 (1984)].

[4] K. van Bibber et al., Phys. Rev. D 39, 2089 (1989).

[5] R. J. Creswick et al., Phys. Lett. B 427, 235 (1998).

[6] J. N. Bachall, M. H. Pinsonneault and S. Basu, Astrophys. J. 555, 990 (2001).

[7] D. M. Lazarus et al., Phys. Rev. Lett. 69, 2333 (1992).

[8] S. Moriyama et al., Phys. Lett. B 434, 147 (1998).

[9] Y. Inoue et al., Phys. Lett. B 536, 18 (2002).

[10] F. T. Avignone et al., Phys. Rev. Lett. 81, 5068 (1998).

[11] A. Morales et al., Astropart. Phys. 16, 325 (2002).

[12] R. Bernabei et al., Phys. Lett. B 515, 6 (2001).

[13] K. Zioutas et al., Nucl. Instrum. Meth. A 425, 480 (1999).

[14] R. Horvat, M. Krčmar and B. Lakić, Phys. Rev. D 69, 125011 (2004).

[15] G. G. Raffelt, Ann. Rev. Nucl. Part. Sci. 49, 163 (1999). 